\title{
Age and Reason for the First Dental Visit of A Child-A Retrospective and Prospective Study
}

\author{
Subashri.A $A^{1}$ and Vignesh Ravindran ${ }^{2}$ \\ ${ }^{1}$ Saveetha Dental College and Hospitals, Saveetha Institute of Medical \\ and Technical Sciences, Saveetha Unviersity, Chennai - 600077, India
}

${ }^{2}$ Senior lecturer, Department of Pediatric and Preventive Dentistry, Saveetha Dental College and Hospitals,

Saveetha Institute of Medical and Technical Sciences, Saveetha University, Chennai-600077, India

\section{ABSTRACT}

According to AAPD guidelines the first dental visit of a child is within 6 months of age. The need of such early visits is to help dentists to detect and treat incipient lesions, to educate parents about proper oral hygiene maintenance and the importance of diet and fluorides. Moreover the visit helps the child to inspect, analyse, understand and adapt to the dental setup, thereby showing positive dental attitude in future visits. The present study was conducted in the Department of Pedodontics which comprised 2 phases Retrospective and Prospective. Inclusion and Exclusion Criteria were framed. A sample size of 200 (out of 225)for retrospective study and 100 for prospective study was taken by Radomised control trial. The most common age group for the first dental visit is 6-12 years. The most common reason for the first dental visit is pain followed by Dental caries and the least common reason is Habits and other reasons such as mobile tooth and lesson etc. The common age of the child's initial dental visit being at $6-12$ years. Within the limitations of the current study, the most common age of the child's first dental visit was at $6-12$ years. The common reason for seeking aid at the primary visit was found to be pain and dental caries. It is evident that the attention level concerning the importance of the primary dental visit is incredibly low among the study population.

KEY WORDS: AGE, REASON, FIRST VISIT, PREVENTIVE, PARENTS, CHILDREN.

\section{INTRODUCTION}

The first time a child visits a dental clinic is their first dental visit. As per AAPD guidelines of 2009, the recommended age for the first dental visit age was before 12 months of age(Rayner, 2003; Widmer, 2003), which in AAPD guidelines of 2013 was changed to 6 months of age.The need of such early visits is to help dentists

\section{ARTICLE INFORMATION}

*Corresponding Author: vigneshr.sdc@saveetha.com Received 11th June 2020 Accepted after revision 10th August 2020 Print ISSN: 0974-6455 Online ISSN: 2321-4007 CODEN: BBRCBA

Thomson Reuters ISI Web of Science Clarivate Analytics USA and Crossref Indexed Journal

\section{Clarivate}

NAAS Journal Score 2020 (4.31) SJIF: 2020 (7.728)

A Society of Science and Nature Publication,

Bhopal India 2020. All rights reserved.

Online Contents Available at: http//www.bbrc.in/

Doi: http://dx.doi.org/10.21786/bbrc/13.7/22 to detect and treat incipient lesions, to educate parents about proper oral hygiene maintenance and importance of diet and fluorides. Moreover the visit helps the child to inspect, analyse, understand and adapt to the dental setup, thereby showing positive dental attitude in future visits. The visit also helps for anticipatory guidance related to the child's craniofacial and dental development. Various literature studies have revealed increased prevalence of Early Childhood Caries in developed and developing countries(Al-Shalan, 2003). The age at which the child first visits the dental clinic and reason for it varies and depends on many factors such as parent's previous dental experience, level of knowledge and socio economic status. Planning and creating awareness about the importance of early visits play a very important role in preventing

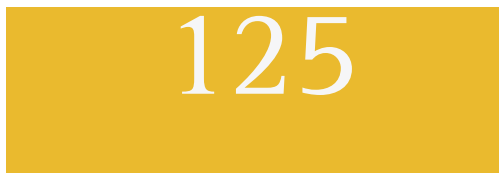


invasive dental procedures. Parents act like mentors and play a vital role in a child's oral health care.

The goals of this early visit are to establish a dental home for the infant, prevent ECC and introduce healthy habits that can be sustained. This visit should include an oral examination, risk assessment and anticipatory guidance. ECC is a highly prevalent chronic condition among children, specifically those with unmet healthcare needs and low socioeconomic status. The prevalence of dental caries among children is of epidemic proportions and ranges between $11.2 \%$ and $48.0 \%$ across the country. While the need for dental treatment among children is high, there is low and delayed utilisation of dental services until oral symptoms such as pain appear and persist. This observed oral health profile of the child is determined by many factors, and the most important of them include the lack of a useful model of dental healthcare aimed at preventive treatment and the low awareness of healthy behaviour among parents.

Our department is passionate about child care, we have published numerous high quality articles in this domain over the past 3 years (Govindaraju et al., 2017a, 2017b; Jeevanandan et al., 2019; Jeevanandan and Govindaraju, 2018; Nair et al., 2018; Panchal et al., 2017; Veerale Panchal et al., 2019; V. Panchal et al., 2019; Ramadurai et al., 2019; Ramakrishnan et al., 2019; Ravikumar et al., 2017, 2018, 2019; Ravindra et al., 2018, 2019; Samuel et al., 2020; Subramanyam et al., 2018; Vignesh et al., 2019; Vishnu Prasad et al., 2018). With this inspiration we planned to pursue our research with the aim to know the average age at which parents first seek dental care for their children and also to find the reasons for the first dental visit of the children in a private dental institute in Chennai.

\section{MATERIAL AND METHODS}

The present study was conducted in the Department of Pedodontics. This study consisted of 2 phases (retrospective and prospective). The inclusion criteria for retrospective phase was children below 17 years of age, no previous history of dental visits and complete cases records. The exclusion criteria were insufficient details in case records, previous history of dental treatment and any missing records of in DMFT/def index. The inclusion criteria for prospective study was children attending the private dental institute for dental treatment, children below 17 years of age and no previous history of dental visit. Exclusion criteria were children with dental emergencies to prevent delay in emergency management.

Retrospective study: From the Department of Pediatric and Preventive Dentistry, patient records from Records Management System were used for the retrospective study. A total of 225 records were taken between November 2018 to December 2018 and evaluated. Twenty five records which did not have adequate details regarding the cause of the first visit were eliminated. A total of 200 record details were considered for the study and data were entered in a data sheet. Then the patients were divided into three age groups, 1) 3-6 years 2) 6-12 years 3) 12-17 years. The following age groups were selected based on dentition present 1) Primary dentition only (3-6) 2) Mixed dentition stage (6-12) 3) Permanent dentition only (12-17). The reasons for their visit were also divided into various categories such as

$\begin{array}{ll}\text { 1. } & \text { General checkup } \\ \text { 2. } & \text { School camp } \\ \text { 3. } & \text { Pain/ sensitivity } \\ \text { 4. } & \text { Malocclusion } \\ \text { 5. } & \text { Dental Caries } \\ \text { 6. } & \text { Trauma } \\ \text { 7. } & \text { Habits } \\ \text { 8. } & \text { Missing/extra tooth } \\ \text { 9. } & \text { Bad breath } \\ \text { 10. } & \text { Others (cleft lip \& palate, mobile tooth, soft }\end{array}$
tissue lesions, other reasons not mentioned below)

Prospective study: A chief investigator in the Department of Pediatric and Preventive Dentistry screened 225 children who were screened by the investigator who visited the dental institute during January 2019 to February 2019. Based on the age, the children were divided into one of the three categories as mentioned in retrospective study. Based on the chief complaint they were categorised into one of the above mentioned reasons. The age and the reason for the visit were enquired and recorded and results were noted in percentages.

Statistical analysis: The collected data were recorded in MS Excel software and subjected to statistical analysis using SPSS software (IBM Corp) version 23.0. Chi-square test was used to compare the age and reasons and p-value of less than 0.05 was considered statistically significant.

\section{RESULTS AND DISCUSSION}

Retrospective study: Percentage distribution of participants in retrospective study based on age. $25.37 \%$ of the participants belonged to 3-6 years, $39.51 \%$ of them were $7-11$ years, $35.12 \%$ of them were $12-16$ years (Figure 1). Percentage distribution of participants in retrospective study based on reason of first dental visit. 8.29\% of the participants reported for general checkup, $13.66 \%$ of them school camp, $25.17 \%$ of them pain, $8.78 \%$ of them due to malocclusion, $17.07 \%$ of them due to caries, $4.39 \%$ of them due to trauma, 3.90\% of them due to habits, $7.32 \%$ of them due to missing, $5.85 \%$ of them due to bad breath, 5.37\% of them due to other reasons (Figure 2). Association between the age and reason of first dental visit of a child. Chi-square test was done and association between the age and reason of first dental visit was found to be statistically significant. (Pearson Chi-Square: 30.27; p-value-0.01). $8.29 \%$ of the participants from $3-6$ years, $8.78 \%$ of the participants from 7-11 years of age and $8.29 \%$ of the participants from 12-16 years of age reported pain as the reason for their first dental visit. Pain followed by caries, school camp and malocclusion were the most common 
reasons for first dental visit according to retrospective study (Figure 3).

Figure 1: Pie diagram represents the percentage distribution of participants in retrospective study based on age. $25.37 \%$ of the participants belonged to 3-6 years (white), 39.51\% of them were 7-11 years (grey), 35.12\% of them were 12-16 years (black)

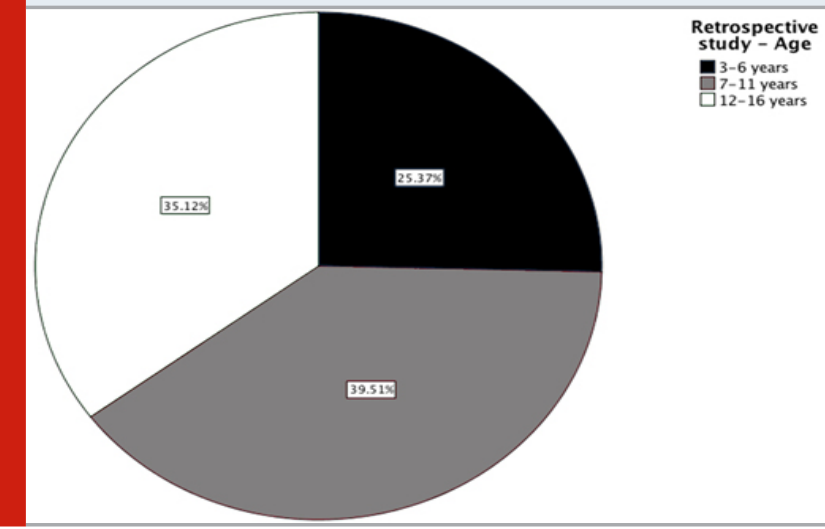

Figure 2: Pie diagram represents the percentage distribution of participants in retrospective study based on reason of first dental visit. 8.29\% of the participants reported for general checkup, (light blue), $13.66 \%$ of them school camp (red), 25.17\% of them pain (green), 8.78\% of them due to malocclusion (orange), $17.07 \%$ of them due to caries (yellow), 4.39\% of them due to trauma (turquoise), 3.90\% of them due to habits (pink), 7.32\% of them due to missing (purple), 5.85\% of them due to bad breath (light green), $5.37 \%$ of them due to other reasons (blue)
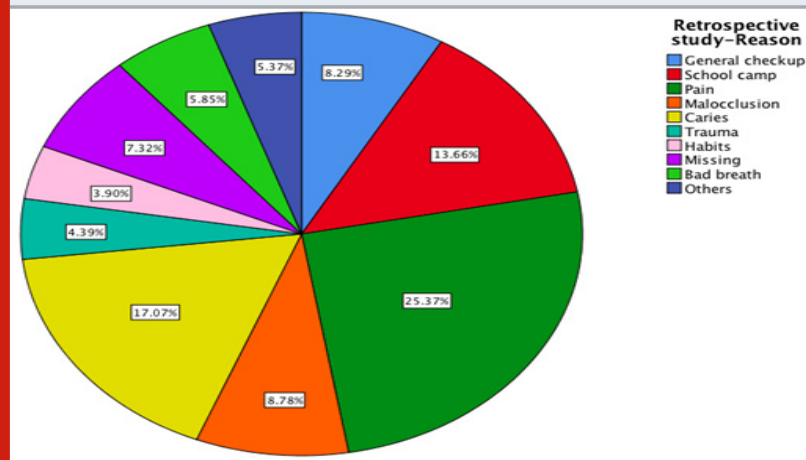

Prospective study: Percentage distribution of participants in prospective study based on age. 25.33\% of the participants belonged to 3-6 years (blue), 37.78\% of them were 7-11 years (red), 36.89\% of them were 12-16 years (Figure 4). Percentage distribution of participants in prospective study based on reason of first dental visit. $7.56 \%$ of the participants reported for general checkup, $12 \%$ of them school camp, $34.22 \%$ of them pain, $6.22 \%$ of them due to malocclusion, $20.89 \%$ of them due to caries, $4 \%$ of them due to trauma, $2.22 \%$ of them due to habits, $4.89 \%$ of them due to missing, $4.44 \%$ of them due to bad breath, 3.56\% of them due to other reasons (Figure 5).
Figure 3: Bar graph depicting the association between the age and reason of first dental visit of a child. $\mathrm{X}$-axis represents the age distribution of the participants and Y-axis represents the number of participants. $8.29 \%$ of the participants from 3-6 years, 8.78\% of the participants from 7-11 years of age and 8.29\% of the participants from 12-16 years of age reported pain as the reason for their first dental visit. Pain followed by caries, school camp and malocclusion were the most common reasons for first dental visit according to retrospective study, which was statistically significant. (Chi-square test; Pearson ChiSquare: 30.27 ; p-value-0.01)

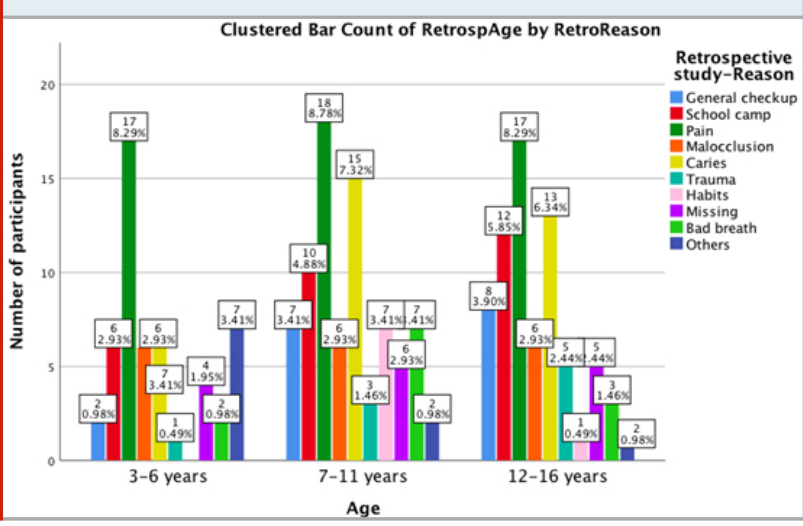

Figure 4: Pie diagram represents the percentage distribution of participants in prospective study based on age. $25.33 \%$ of the participants belonged to 3-6 years (white), 37.78\% of them were 7-11 years (grey), 36.89\% of them were 12-16 years (black)

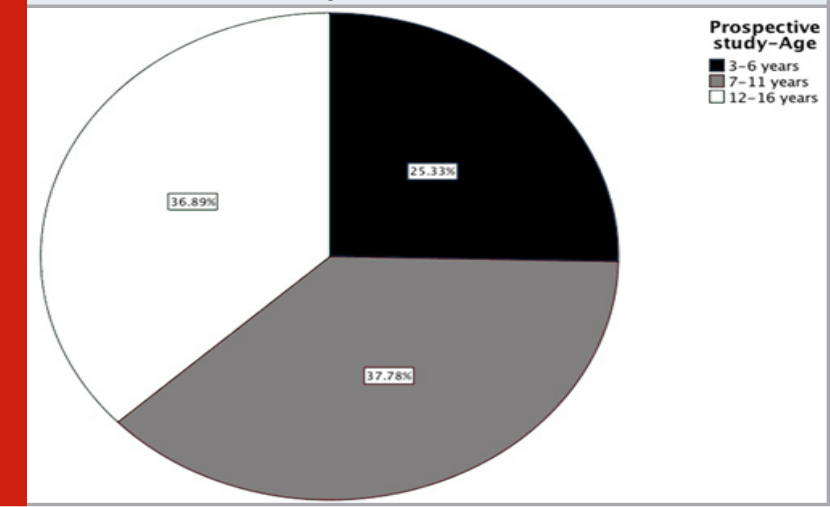

Association between the age and reason of first dental visit of a child. Chi-square test was done and association between the age and reason of first dental visit was found to be statistically significant. (Pearson Chi-Square: 28.25; p-value-0.02). 9.78\% of the participants from 3-6 years, $12 \%$ of the participants from 7-11 years of age and $12.44 \%$ of the participants from 12-16 years of age reported pain as the reason for their first dental visit. Pain followed by caries, trauma and school camp were the most common reasons for first dental visit according to prospective study (Figure 6).

Parents play a key role/ responsible for maintenance of their child's oral hygiene. Their knowledge and 
attitude towards dentistry reflects in their child's oral hygiene. Most of the parents still take their children to the dental practitioner for curative reasons and not for preventive reasons. A huge barrier still exists, that needs to be overcome so as to reach a large-scale reduction in the incidence of dental caries among the developing generation. Thus it's essential to observe these lesions early and intercept them and also to prevent any future occurrence. Several national associations suggest that initial dental visit for a child ought to be by the age of one year or as shortly once the first tooth erupts.The more a child's first dental visit is delayed, the more chances that he or she is to develop serious dental issues that would deteriorate the child's overall health. Caries that are left, Undetected and untreated will cause infection followed by pain, which may eventually stop children from food intake, sleeping, and pursuing their day to day activities, thereby hampering the overall well being of the child. All this would finally end up in an expensive dental treatment or even loss of teeth. All these consequences could have been overcome by one single dental visit at the right time.

Figure 5: Pie diagram represents the percentage distribution of participants in prospective study based on reason of first dental visit. $7.56 \%$ of the participants reported for general checkup, (light blue), 12\% of them school camp (red), 34.22\% of them pain (green), $6.22 \%$ of them due to malocclusion (orange), $20.89 \%$ of them due to caries (yellow), $4 \%$ of them due to trauma (turquoise), $2.22 \%$ of them due to habits (pink), $4.89 \%$ of them due to missing (purple), 4.44\% of them due to bad breath (light green), $3.56 \%$ of them due to other reasons (blue)

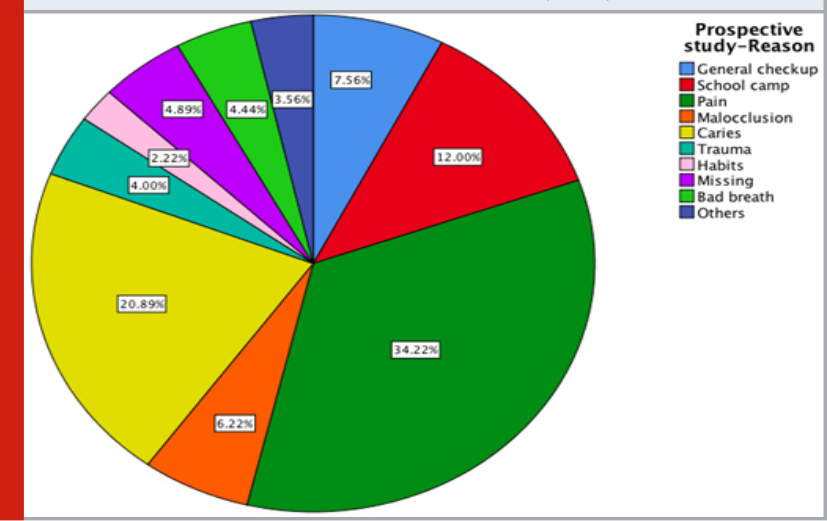

Age of first dental visit: Most of the children within the current study had their first dental visit between the ages of 6 and 12 years. Similarly, a study done in Saudi society believed that dental visitation before the age of one year was inappropriate, with the majority favoring an age range of 3-6 years for the first dental visit(Baghdadi, 2014).Perceptions regarding the appropriate age for initial dental visitation throughout the globe are remarkably diverse; the observed age vary is 2-5 years(van Palenstein Helderman et al., 2006). Older age ranges of 6-12 years(Nainar et al., 2003) and 7-11 years(Baliga, 2019) were observed from Asian countries. The results of those previous studies clearly demonstrate parents' universal reluctance to require their infants to go to the dental practitioner at the young age recommended by the dental academics.

Figure 6: Bar graph depicting the association between the age and reason of first dental visit of a child. $\mathrm{X}$-axis represents the age distribution of the participants and Y-axis represents the number of participants. 9.78\% of the participants from 3-6 years, $12 \%$ of the participants from 7-11 years of age and $12.44 \%$ of the participants from 12-16 years of age reported pain as the reason for their first dental visit. Pain followed by caries, trauma and school camp were the most common reasons for first dental visit according to prospective study, which was statistically significant. (Chi-square test; Pearson Chi-Square value: 28.25; p-value-0.02)

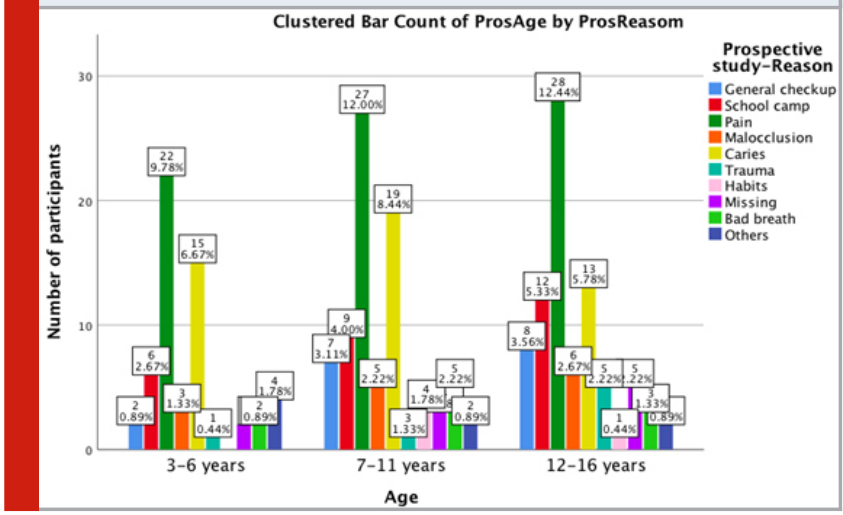

According to Fuzre and Basso, the primary dental visit should be around the fourth month of intrauterine life(Furze and Basso, 2003). Throughout this visit by the expectant mother, the medical practitioner has a chance to elucidate the importance of the dental visit at six months mature, educate the mother on eruption of teeth and preventive procedures, and to supply parent messages(Mileva and Kondeva, 2010). Nainar and Straffon in their study showed that the primary dental visit should be performed at one year age for all children from an occasional low socioeconomic background(Nainar et al., 2003). Study showed that within the US about thirty two percentage of children aged 2 to 4 years had a dental visit within the past twelve months. Slayton et al. in their Iowa halide Study reported that among children between birth to three years about two of the parents reported that their kid had a dental visit by one year of age(Gorito et al., 2019). A study by Pierce and coworkers, showed that medical aid suppliers tend to under- refer, and solely seventieth percentage of children with proof of dental illness received a referral. A study by Cunha in a Brazilian population showed that, following the associate awareness program applied by the Baby Clinic of the grad school of Aracatuba, the foremost common reported reason for infants to gain attention was the parents' desire for orientation and bar(dela Cruz et al., 2004).

Reason for first dental visit: If not for pain and alternative dental emergencies (e.g., trauma and infection), most 
participants during this study might not have visited the dental clinics at the recorded ages. Alternative studies conducted in Saudi Arabia have additionally documented late exposure to aid, with considerable percentages of parents denied for dental visitation if their children weren't in pain . Results of alternative studies conducted in several places replicate identical perspectives, with pain reported as the dominant issue prompting parents to visit for initial dental appointments for their children.

Present study showed a low awareness level in the population, as the majority of the children were brought for the first dental visit at 6 to 12 years of age and the commonest reason for seeking dental care was pain and dental caries. It is also evident that parents bring their child for a dental visit only when the disease is moderate to severe. In the present study, low percentages of parents wanted initial aid for their children for preventive reasons, like check-up or fluoride application. These results demonstrate a transparent lack of dental data and unknowingness of the importance of dentition among folks of the study participants. Baghdadi in 2014 stated that similar attitudes among people in Saudi society, showing under-recovery of the role that teeth-particularly primary teeth-play within the general health and well-being of their children, further as a scarcity of data relating to the suitable time for a child's first dental visit (Bagramian et al., 2009). It is also good to notice $15 \%$ of participants reported due to referral from camps conducted in schools. This proves that practitioners/ educational institutions promote dental awareness among school children.

\section{CONCLUSION}

Within the limitations of the current study, the most common age of the child's first dental visit was at $6-12$ years. The common reason for seeking aid at the primary visit was found to be pain and dental caries. It is evident that the attention level concerning the importance of the primary dental visit is incredibly low among the study population.

\section{REFERENCES}

Al-Shalan TA (2003) Factors Affecting Saudi Parents' Perception of their Children's First Dental Visit. The Journal of Contemporary Dental Practice. DOI: 10.5005/ jcdp-4-4-54.

Baghdadi Z (2014) Improving Oral Health Status of Children in Tabuk, Saudi Arabia. Dentistry Journal. DOI: $10.3390 /$ dj2010022.

Bagramian RA, Garcia-Godoy F and Volpe AR (2009) The global increase in dental caries. A pending public health crisis. American journal of dentistry 22(1): 3-8.

Baliga M (2019) Child's first dental visit in India: A reappraisal. Journal of Indian Society of Pedodontics and Preventive Dentistry. DOI: 10.4103/jisppd. jisppd_195_19.

dela Cruz GG, Rozier RG and Slade G (2004) Dental screening and referral of young children by pediatric primary care providers. Pediatrics 114(5): e642-52.

Furze H and Basso M (2003) The first dental visit: an Argentine point of view. International journal of paediatric dentistry / the British Paedodontic Society [and] the International Association of Dentistry for Children 13(4): 266-268.

Gorito V, Monjardino T, Azevedo I, et al. (2019) 0C6 Pediatric pain at 10 years old in a portuguese birth cohort - reported by children. Abstracts. DOI: 10.1136/ archdischild-2019-epa.6.

Govindaraju L, Jeevanandan G and Subramanian EMG (2017a) Comparison of quality of obturation and instrumentation time using hand files and two rotary file systems in primary molars: A single-blinded randomized controlled trial. European journal of dentistry 11(3): 376-379.

Govindaraju L, Jeevanandan G and Subramanian EMG (2017b) Knowledge and practice of rotary instrumentation in primary teeth among indian dentists: A questionnaire survey. Journal of International Oral Health 9(2): 45.

Jeevanandan G and Govindaraju L (2018) Clinical comparison of Kedo-S paediatric rotary files vs manual instrumentation for root canal preparation in primary molars: a double blinded randomised clinical trial. European archives of paediatric dentistry: official journal of the European Academy of Paediatric Dentistry 19(4): 273-278.

Jeevanandan G, Ganesh S and Arthilakshmi (2019) Kedo file system for root canal preparation in primary teeth. Indian journal of dental research: official publication of Indian Society for Dental Research 30(4): 622-624. Mileva S and Kondeva V (2010) Age at and Reasons for the First Dental Visit. Folia Medica. DOI: 10.2478/ v10153-010-0018-x.

Nainar SMH, Hashim Nainar SM and Straffon LH (2003) Targeting of the Year One dental visit for United States children. International Journal of Paediatric Dentistry. DOI: 10.1046/j.1365-263x.2003.04602.x.

Nair M, Jeevanandan G, Vignesh R, et al. (2018) Comparative evaluation of post-operative pain after pulpectomy with k-files, kedo-s files and mtwo files in deciduous molars -a randomized clinical trial. Brazilian Dental Science 21(4): 411.

Panchal V, Gurunathan D and Shanmugaavel AK (2017) Smartphone application as an aid in determination of caries risk and prevention: A pilot study. European journal of dentistry 11(4): 469-474.

Panchal Veerale, Jeevanandan G and Subramanian E (2019) Comparison of instrumentation time and obturation quality between hand K-file, H-files, and rotary Kedo-S in root canal treatment of primary teeth: A randomized controlled trial. Journal of the Indian Society of Pedodontics and Preventive Dentistry 37(1): 75-79.

Panchal V., Jeevanandan G and Subramanian EMG 
(2019) Comparison of post-operative pain after root canal instrumentation with hand K-files, H-files and rotary Kedo-S files in primary teeth: a randomised clinical trial. European archives of paediatric dentistry: official journal of the European Academy of Paediatric Dentistry 20(5): 467-472.

Ramadurai N, Gurunathan D, Samuel AV, et al. (2019) Effectiveness of 2\% Articaine as an anesthetic agent in children: randomized controlled trial. Clinical oral investigations 23(9): 3543-3550.

Ramakrishnan M, Dhanalakshmi R and Subramanian EMG (2019) Survival rate of different fixed posterior space maintainers used in Paediatric Dentistry - A systematic review. The Saudi dental journal 31(2): 165-172.

Ravikumar D, Jeevanandan G and Subramanian EMG (2017) Evaluation of knowledge among general dentists in treatment of traumatic injuries in primary teeth: A cross-sectional questionnaire study. European journal of dentistry 11(2): 232-237.

Ravikumar D, Gurunathan D, Gayathri R, et al. (2018) DNA profiling of Streptococcus mutans in children with and without black tooth stains: A polymerase chain reaction analysis. Dental research journal 15(5): 334.

Ravikumar D, N S, Ramakrishna M, et al. (2019) Evaluation of McNamara's analysis in South Indian (Tamil Nadu) children between 8-12 years of age using lateral cephalograms. Journal of oral biology and craniofacial research 9(2): 193-197.

Ravindra V, Rekha V, Annamalai S, et al. (2018) A comparative evaluation between dermatoglyphic patterns and different terminal planes in primary dentition. Journal of clinical and experimental dentistry 10(12): e1149-e1154.

Ravindra V, Rekha CV, Annamalai S, et al. (2019) A comparative evaluation between cheiloscopic patterns and the permanent molar relationships to predict the future malocclusions. Journal of clinical and experimental dentistry 11(6): e553-e557.

Rayner JA (2003) The first dental visit: a UK viewpoint. International journal of paediatric dentistry / the British Paedodontic Society [and] the International Association of Dentistry for Children.

Samuel SR, Acharya S and Rao JC (2020) School Interventions-based Prevention of Early-Childhood Caries among 3-5-year-old children from very low socioeconomic status: Two-year randomized trial. Journal of public health dentistry 80(1): 51-60.

Subramanyam D, Gurunathan D, Gaayathri R, et al. (2018) Comparative evaluation of salivary malondialdehyde levels as a marker of lipid peroxidation in early childhood caries. European journal of dentistry 12(1): 67-70.

van Palenstein Helderman WH, Soe W and van 't Hof MA (2006) Risk factors of early childhood caries in a Southeast Asian population. Journal of dental research 85(1): 85-88.

Vignesh R, Sharmin D, Rekha CV, et al. (2019) Management of Complicated Crown-Root Fracture by Extra-Oral Fragment Reattachment and Intentional Reimplantation with 2 Years Review. Contemporary clinical dentistry 10(2): 397-401.

Vishnu Prasad S, Kumar M, Ramakrishnan M, et al. (2018) Report on oral health status and treatment needs of 5-15 years old children with sensory deficits in Chennai, India. Special care in dentistry: official publication of the American Association of Hospital Dentists, the Academy of Dentistry for the Handicapped, and the American Society for Geriatric Dentistry 38(1): 58-59.

Widmer R (2003) The first dental visit: an Australian perspective. International journal of paediatric dentistry / the British Paedodontic Society [and] the International Association of Dentistry for Children 13(4): 270. 